\title{
Linkage Mapping for Foliar Necrosis of Soybean Sudden Death Syndrome
}

\author{
Hao-Xun Chang, ${ }^{1}$ Zixiang Wen, ${ }^{2}$ Ruijuan Tan, ${ }^{2}$ Hongxu Dong, ${ }^{3}$ Daniel P. Wickland, ${ }^{4}$ Dechun Wang, ${ }^{2}$ \\ and Martin I. Chilvers $2, \dagger$ \\ ${ }^{1}$ Department of Plant Pathology and Microbiology, National Taiwan University, Taipei 10617, Taiwan \\ ${ }^{2}$ Department of Plant, Soil and Microbial Sciences, Michigan State University, East Lansing, MI 48823, U.S.A. \\ 3 Plant Genome Mapping Laboratory, University of Georgia, Athens, GA 30605, U.S.A. \\ ${ }^{4}$ Department of Health Sciences Research, Mayo Clinic, Jacksonville, FL 32224, U.S.A. \\ Accepted for publication 5 December 2019.
}

\begin{abstract}
Sudden death syndrome (SDS) foliar symptoms consist of foliar chlorosis, foliar necrosis, leaf marginal curling, and premature defoliation, but resistance screening has been evaluated mostly based on the overall SDS foliar severity rather than on a specific foliar symptom. This study generated an $\mathrm{F}_{2}$ population derived from crossing the susceptible variety Sloan and the resistant germplasm line PI 243518, which exhibits resistance to both foliar chlorosis and necrosis. A total of $400 \mathrm{~F}_{2}$ lines were evaluated for foliar chlorosis, foliar necrosis, and overall SDS foliar symptoms, separately. Genotyping-by-sequencing was applied to obtain single nucleotide polymorphisms (SNPs) in the $F_{2}$ population, and

linkage mapping using $135 \mathrm{~F}_{2}$ lines with 969 high-quality SNPs identified a locus on chromosome 13 for foliar necrosis and SDS foliar symptoms. The locus partially overlaps with loci previously reported for SDS on chromosome 13, which is the third time the region from 15.98 to 21.00 Mbp has been reproduced independently and therefore qualifies this locus for a new nomenclature proposed as $R f v 13-02$. In summary, this study generated a new biparental population that enables not only the discovery of a locus for foliar necrosis and SDS foliar symptoms on chromosome 13 but also the potential for advanced exploration of SDS foliar resistance derived from the germplasm line PI 243518.
\end{abstract}

Soybean sudden death syndrome (SDS) has been a top-ranking soybean disease in the United States for decades (Koenning and Wrather 2010; Wrather and Koenning 2009) and it has been ranked as one of the top five yield-limiting diseases in the northern United States and in Ontario, Canada, from 2010 to 2014 (Allen et al. 2017). The average annual loss caused by SDS was documented at $\$ 133.3$ million from 1988 to 2010, and the highest loss was reported to be $\$ 669.2$ million in 2010 (Navi and Yang 2016). The major casual pathogen of soybean SDS in the United States is Fusarium virguliforme, which infects soybean roots and causes root rot and necrosis. $F$. virguliforme has been shown to inhabit roots and soils, and the pathogen cannot be isolated or even rarely detected using quantitative PCR on the stem and leaves of soybean (Hartman et al. 2015 ). However, the phytotoxins of $F$. virguliforme cause foliar symptoms at the late growth stage or flowering stage, where severe interveinal chlorosis and necrosis suddenly show up in fields with high disease pressure. Different approaches such as agronomic practices, fungicide seed treatments early detection by remote sensing, and risk assessments have been studied to optimize SDS management (Herrmann et al. 2018; Kandel et al. 2016a, b, 2019a, b; Roth et al. 2019). In addition, the development of an SDSresistant variety remains a key strategy for disease management. Characterization of SDS resistance genes in soybean relies on mapping strategies such as biparental linkage mapping and genome-wide association studies (GWASs) (Luckew et al. 2017;

${ }^{\dagger}$ Corresponding author: M. I. Chilvers; chilvers@msu.edu

Funding: This project was supported by the North Central Soybean Research Program and Michigan Soybean Promotion Committee (to M. I. Chilvers) and Michigan State University Project GREEEN (grant GR19-059 to H.-X. Chang and M. I. Chilvers).

*The $\boldsymbol{e}$-Xtra logo stands for "electronic extra" and indicates that two supplementary figures are published online.

The author(s) declare no conflict of interest.

(c) 2020 The American Phytopathological Society
Wen et al. 2014; Zhang et al. 2015). Because only a few germplasm lines exhibiting SDS resistance were found in the U.S. Department of Agriculture (USDA) germplasm collection (Hartman et al. 2015; Mueller et al. 2002), GWASs become relatively difficult because rare variants may be excluded by the minor allele frequency cutoff (Chang et al. 2016b) and the phenotypic distribution may be heavily skewed toward susceptibility in a GWAS diversity panel. On the other hand, biparental populations derived from crossing a susceptible and a resistant variety overcome these issues, but the development of biparental populations is time-consuming, labor intensive, and the SDS resistance sources can only be explored in the genetic background of the resistant parent. To date, only a few resistant germplasm lines or varieties have been used to create populations.

The most studied biparental population is the cross between the partially resistant variety Forrest (PI 548655) and the susceptible variety Essex. Several studies using this population identified quantitative trait loci (QTLs) on chromosomes 3, 6, 8, 11, 13, 16, 18, and 20 (Abdelmajid et al. 2007; Chang et al. 1996; Hnetkovsky et al. 1996; Kassem et al. 2006; Iqbal et al. 2001; Meksem et al. 1999; Njiti et al. 2002; Prabhu et al. 1999; Yuan et al. 2012). Studies using other populations have uncovered additional QTLs. For example, the biparental population generated by crossing the partially resistant variety Hartwig (PI 543795) and the susceptible variety Flyer identified QTLs on chromosomes 6, 17, and 18 (Kazi et al. 2008). The biparental population generated by crossing the partially resistant variety Pyramid (PI 512039) and the susceptible variety Douglas identified QTLs on chromosomes 3, 6, and 18 (Njiti et al. 2002). In addition, the partially resistant germplasm line PI 438489B was crossed with the susceptible variety Hamilton and the study identified QTLs on chromosomes 1, 2, 3, 4, 6, 8, 10, 11, 18, and 19 (Abdelmajid et al. 2012). The partially resistant variety MD 96-5722 (a.k.a. 'Monocacy') was crossed with the susceptible variety Spencer, and QTLs were identified on chromosomes 1, 3, 5, 6, 9, 10, 13, 14, 15, and 16 (Anderson et al. 2015).

Studies using a population crossed by the partially resistant variety LD01-5907 and the susceptible variety GD2422 identified 
QTLs on chromosomes 4, 8, 12, and 18 (Tan et al. 2018) and using a population derived from the cross of the partially resistant variety E07080 and the susceptible variety U01-390489 identified QTLs on chromosomes 6, 7, 9, 17, 18, and 20 (Tan et al. 2019). Another study identified a significant marker on chromosome 10 using a population generated by the partially resistant variety Ripley crossed with the susceptible variety LD02-4485. In addition, significant markers on chromosomes 1 and 18 were found using a population generated by the partially resistant germplasm line PI 507531 and the susceptible variety Spencer and three populations using a partially resistant germplasm line PI 567374 and three susceptible varieties (LD02-4485, LD01-5907, and LD00-3309) all led to the identification of cqSDS-001 on chromosome 17, providing significant confidence for this locus, which has become the first locus confirmed by the Soybean Genetics Committee (Brzostowski et al. 2018).

Other than screening SDS resistance in fields, a few studies have applied the phytotoxic culture filtrate of $F$. virguliforme to identify resistance genes in soybean. For example, the partially resistant variety LS94-3207 and the partially resistant variety LS98-0582 were separately crossed with the susceptible variety A95-684043, and resistance screening and mapping in these populations identified QTLs on chromosomes 2, 5, 8, 13, 16, and 20 and QTLs on chromosomes $6,7,8,9,10,13$, and 20, respectively (Swaminathan et al. 2016). These two populations were further screened in the greenhouse against $F$. virguliforme infection and the results identified QTLs on chromosomes 9, 13, 17, 19, and 20 (Swaminathan et al. 2018). Another study reported the loss-ofsusceptibility mechanism for foliar chlorosis, which relies on the double mutation of soybean STAY-GREEN genes. Unfortunately, photosynthesis reduction and leaf fragility were observed in soybean lines containing double mutation in the STAY-GREEN

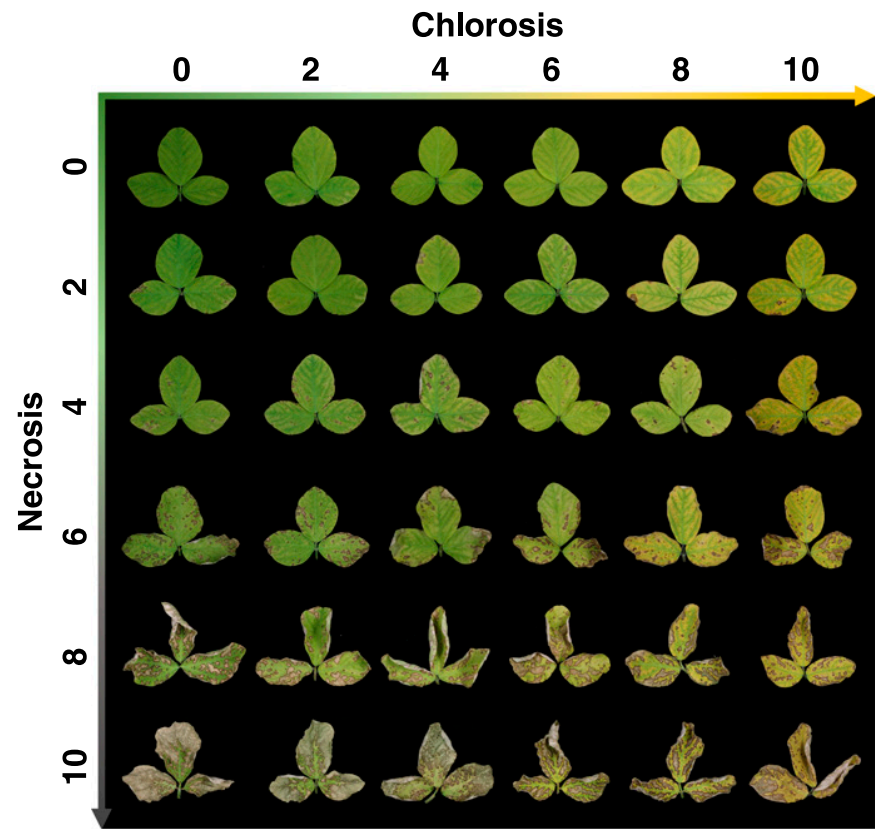

Fig. 1. Phenotyping scale reference for foliar chlorosis and foliar necrosis in the $F_{2}$ population of PI $243518 \times$ Sloan. The first trifoliate of each soybean plant was treated with culture filtrate of Fusarium virguliforme, and the phenotypes were rated and photographed at 8 days postinoculation. The upperleft leaf was the resistant parent PI 243518, which exhibited resistance to both chlorosis and necrosis. The lower-right leaf was the susceptible parent 'Sloan', which exhibited susceptibility to both chlorosis and necrosis. The $\mathrm{F}_{2}$ lines displayed different levels of chlorosis and necrosis. $F_{2}$ lines susceptible to chlorosis may display resistance to necrosis (e.g., upper-right trifoliate), whereas $\mathrm{F}_{2}$ lines resistant to chlorosis may display susceptibility to necrosis (e.g., lower-left trifoliate). Therefore, this ordinal scale reference provides a scale of possible combinations of foliar chlorosis (ordinal scale 0 to 10) and foliar necrosis (ordinal scale 0 to 10 ). genes, which makes these resistant germplasm lines unfavorable in breeding programs (Chang et al. 2019).

Because of the quantitative nature of SDS resistance and the differences in experimental methods, mapping studies for SDS resistance have found more than 100 QTLs and single nucleotide polymorphisms (SNPs) associated with SDS resistance, and only 10 loci are reproducible by three or more studies (Chang et al. 2018). Therefore, research efforts are needed to explore additional sources and understand the molecular mechanism of SDS resistance. In our previous study, the germplasm line PI 243518 was identified to harbor resistance to SDS foliar symptoms (Chang et al. 2019); therefore, the goal of this study was to generate a biparental population for mapping SDS foliar resistance derived from PI 243518. In this study, the resistant germplasm line PI 243518 was crossed to the susceptible variety Sloan to generate an $\mathrm{F}_{2}$ population for screening and mapping foliar chlorosis, foliar necrosis, and the overall SDS foliar symptoms. Genotyping-by-sequencing (GBS) was applied to obtain the genotypes, and $135 \mathrm{~F}_{2}$ lines with high-quality SNPs were included in the linkage mapping, which identified a novel locus on chromosome 13 for foliar necrosis and SDS foliar symptoms.

\section{MATERIALS AND METHODS}

Generation of the $F_{2}$ biparental crossing population. The resistant soybean germplasm line PI 243518 was used as the female parent and the susceptible soybean variety Sloan was used as the male parent in a biparental crossing experiment conducted in the greenhouse at Michigan State University in July 2018. Three pods were regarded as hybrids based on the absence of sepals (Supplementary Fig. S1). Seven seeds from these three pods were confirmed as true $F_{1}$ seeds using the Kompetitive Allele-Specific Polymerase (KASP) chain reaction with seven random-selected allele-specific primers (Supplementary Fig. S2). These seven seeds were propagated in the greenhouse to harvest the $\mathrm{F}_{2}$ population from September 2018 to January 2019.

Phenotyping for foliar chlorosis, foliar necrosis, and SDS foliar symptoms. The stem cutting assay was used for phenotyping foliar necrosis, foliar chlorosis, and SDS foliar symptoms (Xiang et al. 2015). In brief, the culture filtrate of $F$. virguliforme Mont1 grown in potato dextrose broth (PDB) was used as inoculation material throughout the study. F. virguliforme was incubated in $\mathrm{PDB}$ at $25^{\circ} \mathrm{C}$ in darkness for 20 days, and $2.5 \mathrm{ml}$ of the $0.22-\mu \mathrm{m}$ filtered cell-free culture filtrate was added to a $50-\mathrm{ml}$ conical tube containing $47.5 \mathrm{ml}$ of distilled deionized water. The $\mathrm{F}_{2}$ soybean plants were germinated and grown in a plant growth chamber at $25^{\circ} \mathrm{C}, 60 \%$ relative humidity, and a 16-h/day photoperiod with a light intensity of $380 \mathrm{mE} / \mathrm{m}^{2}$. The first trifoliate of each $\mathrm{F}_{2}$ soybean plant was cut and the trifoliate end was immersed in a conical tube prepared with the culture filtrate of $F$. virguliforme. The severity was recorded based on the degree of foliar chlorosis, foliar necrosis, and the overall SDS foliar symptoms (considering chlorosis, necrosis, leaf marginal curling, and defoliation) at $0,2,4,6$, and 8 days postinoculation using an ordinal scale (Fig. 1) (Chang et al. 2016a). Area under the disease progression curves (AUDPCs) were calculated based on the following formula:

$$
\text { Severity }=\sum \frac{d_{i}+d_{i+1}}{2}\left(t_{i+1}-t_{i}\right)
$$

where $d$ is the degree, $t$ is the time, and $i$ is an increment from 0 to 4 (Madden et al. 2007). The correlations of the foliar chlorosis AUDPC, foliar necrosis AUDPC, and SDS foliar symptoms AUDPC were estimated using Pearson's statistic method in the R version 3.4.3 environment.

GBS to acquire SNPs. Genomic DNA of each $\mathrm{F}_{2}$ progeny line was extracted from soybean leaves using the CTAB-chloroformisopropanol method. GBS was conducted according to the protocol 
of Elshire et al. (2011). In brief, each DNA sample was quantified using the Quant-iT Broad-Range dsDNA Assay Kit (Invitrogen/ Thermo Fisher Scientific, Waltham, MA), and $250 \mathrm{ng}$ of DNA was digested by ApeKI (New England Biolabs, Ipswich, MA) at $75^{\circ} \mathrm{C}$ for $3 \mathrm{~h}$. Digested soybean DNA was ligated to $0.1 \mu \mathrm{M}$ of barcoded adaptor and $0.1 \mu \mathrm{M}$ of common adaptor using T4 ligase (New England Biolabs) at $22^{\circ} \mathrm{C}$ for $2 \mathrm{~h}$. All 96 ligated soybean samples were multiplexed for pre-PCR gel extraction using the Monarch DNA Gel Extraction Kit (New England Biolabs) targeting 200- to 500-bp DNA fragments. These pre-PCR gelextracted DNA fragments were used as a template for 16 cycles of PCR. A post-PCR gel extraction was conducted to exclude primer dimers and to further purify amplicon fragments ranging from 200 to $500 \mathrm{bp}$. Post-PCR gel-extracted DNA was quality assessed using the 2100 BioAnalyzer (Agilent, Santa Clara, CA) before subjecting the sample to one lane of 150-bp single end sequencing using the NextSeq500 platform (Illumina, San Diego, CA) at the Research Technology Support Facility at Michigan State University.

The raw reads of 96 multiplexed samples generated by each lane of NextSeq500 were preprocessed using Cutadapt version 2.3 (Martin 2011), including removing reads with sequencing quality below a Phred score of 30 and trimming the adaptor sequences at the $5^{\prime}$ and $3^{\prime}$ ends. The quality-controlled reads were subjected to the TASSEL version 5.0 GBS pipeline version 2.0 for SNP calling at a minimal score of 20 (Bradbury et al. 2007; Wickland et al. 2017). The preliminary SNP matrix was inputted into the R environment to identify polymorphic SNPs between two parents, and the polymorphic SNPs were further tested for segregation distortion using R package "qtl" version 1.44-9. SNPs receiving adjusted $P$ values below the Bonferroni correction were excluded. In addition, $\mathrm{F}_{2}$ progeny lines with $>20 \%$ missing SNPs and SNPs with $>20 \%$ absence in the population were excluded. Subsequently, duplicated $\mathrm{F}_{2}$ progeny lines and duplicated markers with $90 \%$ or higher similarity were excluded. The quality-controlled SNPs were kept for linkage group (LG) construction.

LG construction and linkage mapping. The qualitycontrolled SNPs were subjected to LG construction using the MSTmap in a single LG criterium, recombinant inbred line (RIL) at generation 2 for population type, and a genetic mapping function of Kosambi with no mapping distance threshold adjusted to $100 \mathrm{cM}$ (Wu et al. 2008). The genetic distances were incorporated in linkage mapping using R packages 'qtl' and 'qtl2' (version 0.20) (Broman et al. 2003, 2019). Using the $\mathrm{R}$ package 'qtl', the pairwise recombination fraction was estimated and QTL mapping using the Haley-Knott regression method (Feenstra et al. 2006) was applied at error probability at 0.05 . In addition, the significance of these interval mapping methods was determined by performing permutation tests with 10,000 replicates, and the QTL peak as well as the confidence intervals were estimated by the Bayes credible function. The genotype of the most significant SNP was estimated by the maximum marginal probability at 0.95 using the 'qt12' package. The significance of additive effect was estimated by converting the $\mathrm{AA}, \mathrm{AB}$, and $\mathrm{BB}$ genotypes to 2,1 , and 0 as an explanatory variable in a linear model and using the square root-transformed phenotypes as the response variables to reduce violation in normality assumption. The significance of dominant effect was estimated by converting the $\mathrm{AA}$ and $\mathrm{AB}$ genotypes into the $A$ allele and $\mathrm{BB}$ genotypes into the $a$ allele as an explanatory variable in a $t$ test and using the square root-transformed phenotypes as the response variables to reduce violation in normality assumption.

\section{RESULTS}

Phenotyping foliar chlorosis, foliar necrosis, and SDS foliar symptoms in the $F_{2}$ population of resistant PI 243518 and susceptible 'Sloan'. Germplasm line PI 243518 was highly resistant to SDS foliar symptoms in screening SDS resistance using
A

$F_{2}$ lines $=400$

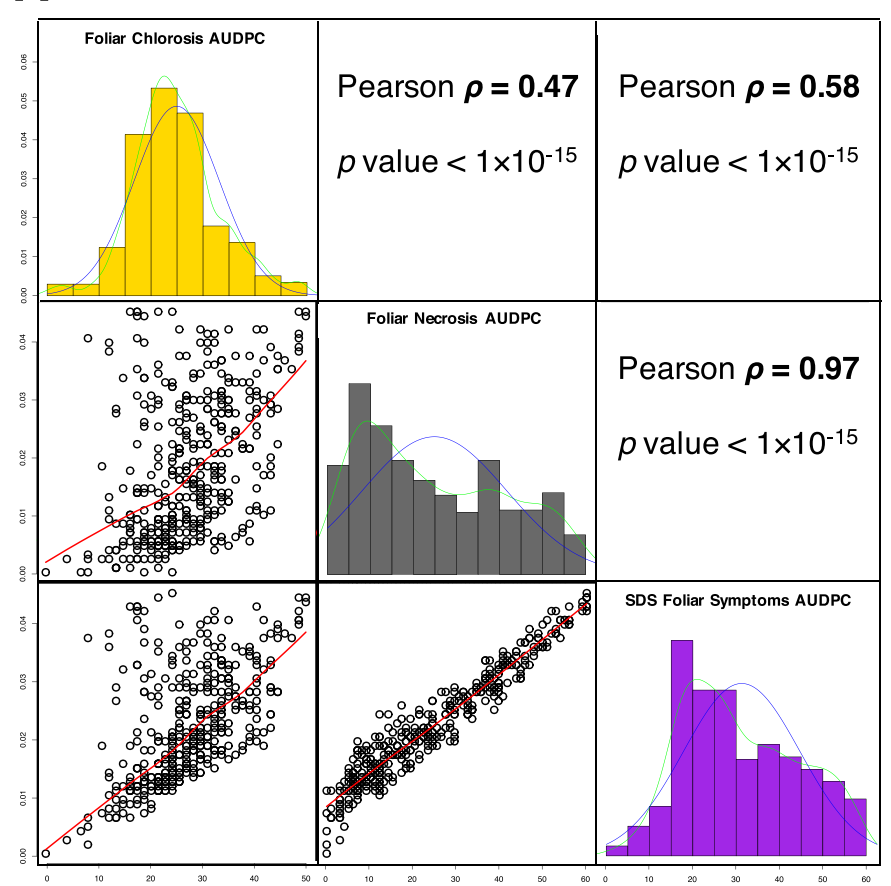

B

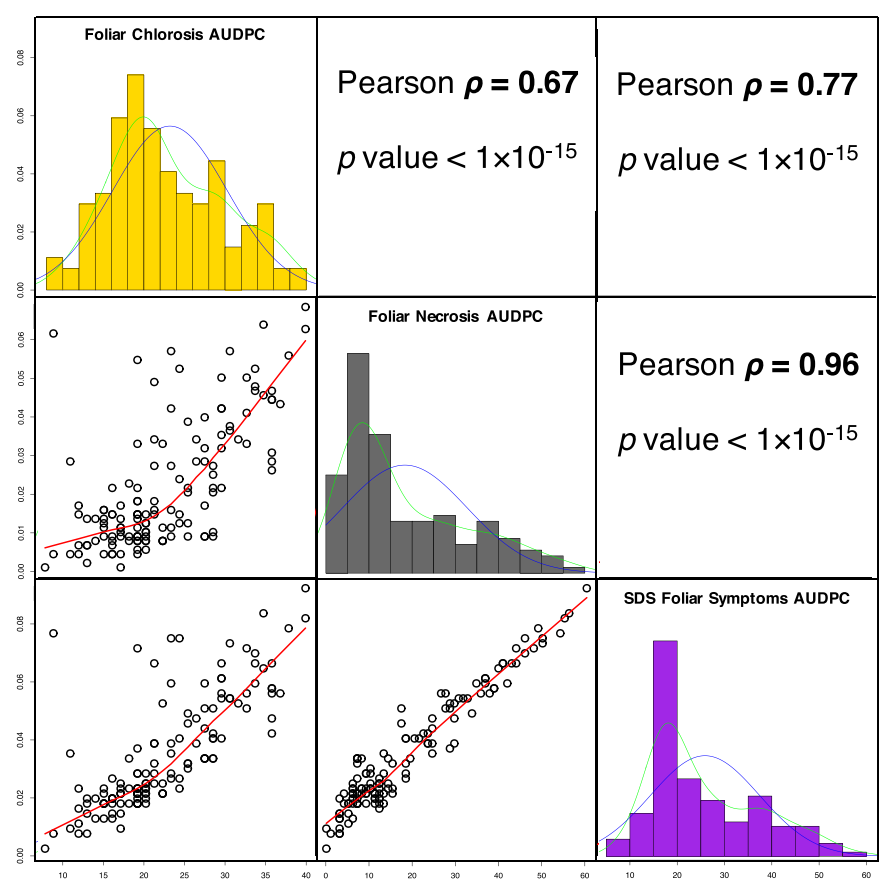

Fig. 2. Phenotypic distributions and pairwise correlations of the foliar chlorosis area under disease progression curve (AUDPC), foliar necrosis AUDPC, and sudden death syndrome (SDS) foliar symptoms AUDPC. The AUDPCs at 8 days postinoculation of $\mathrm{F}_{2}$ lines were analyzed and are shown in the graphs. Yellow distributions indicate the phenotypic observation of the foliar chlorosis AUDPC. Gray distributions indicate the phenotypic observation of the foliar necrosis AUDPC. Purple distributions indicate the phenotypic observation of the overall SDS foliar symptoms AUDPC. The green curves indicate the regression curve based on the observation, whereas the blue curves indicate the theoretical normal distribution. A, Distributions and correlations among the $400 \mathrm{~F}_{2}$ lines. Pairwise correlations were all significant for the three phenotypic ratings. The phenotypic distribution of the foliar chlorosis AUDPC was approximately normal but not for the SDS foliar necrosis AUDPC or the SDS foliar symptoms AUDPC. B, Distributions and correlations among the $135 \mathrm{~F}_{2}$ lines. 
the USDA soybean germplasm lines (Chang et al. 2016b, 2019). The first trifoliate of PI 243518 remained green and healthy for 7 to 10 days post-treatment with the $F$. virguliforme phytotoxic culture filtrate, whereas the first trifoliate of most soybean germplasm lines displayed different levels of foliar chlorosis or necrosis. Seven $\mathrm{F}_{1}$ seeds from three pods were obtained from PI $243518 \times$ 'Sloan' in the greenhouse (Supplementary Fig. S1). The DNA of these seven $\mathrm{F}_{1}$ seedlings was extracted, and seven random primers were used to
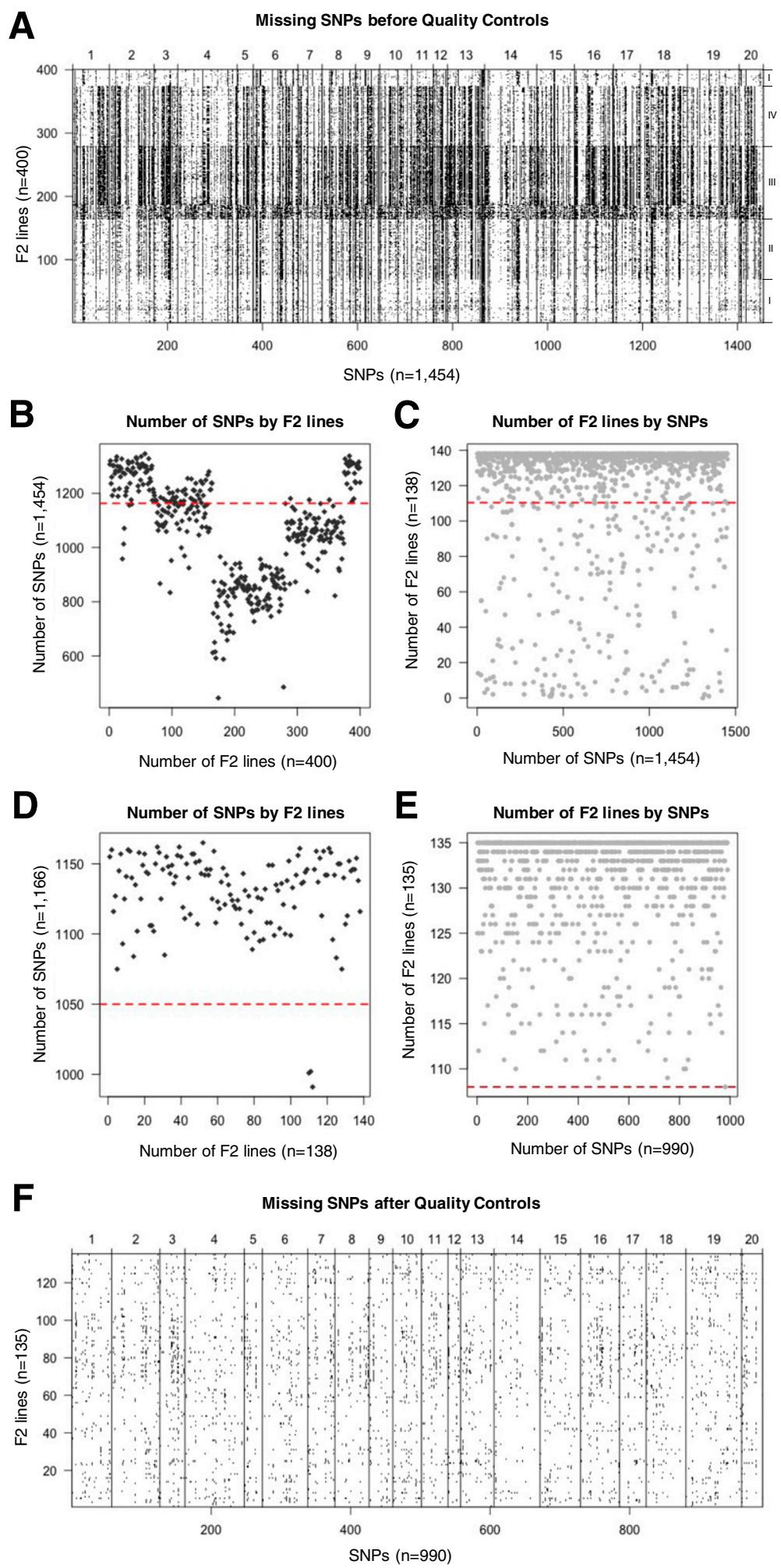

Fig. 3. Single nucleotide polymorphism (SNP) calling and quality controls. A, The dark dots represent missing SNPs in the $400 \mathrm{~F}_{2}$ lines of PI $243518 \times$ 'Sloan'. Many $\mathrm{F}_{2}$ lines had $>20 \%$ missing SNPs and many SNPs had $>20 \%$ absence in the population. $\mathbf{B}$, The dark gray diamonds represent $400 \mathrm{~F}_{2}$ lines, and $\mathrm{F}_{2}$ lines with $<20 \%$ SNPs (above dashed line) were retained for downstream analyses. C, The light gray dots represent SNPs and the 1,454 SNPs absent from $<20 \%$ of the population (above the red dash line) were retained for downstream analyses. D, The dark gray diamonds represent $138 \mathrm{~F} 2$ lines, and three outliers with $<1,050$ SNPs were filtered out from the downstream analyses. $\mathbf{E}$, After removing duplicated SNPs and $\mathrm{F}_{2}$ lines with $90 \%$ or higher similarity, there were 990 SNPs retained after the quality control. The light gray dots represent 990 SNPs, and the red dash line indicates the $80 \%$ population size threshold (135 individuals). F, The overall quality controls reduced the mapping population from 400 to $135 \mathrm{~F}_{2}$ lines and from 1,454 to 990 SNPs for constructing linkage groups. 
confirm that the seven $F_{1}$ seeds were heterozygous using KASP (Supplementary Fig. S1). The seven $\mathrm{F}_{1}$ seeds were subsequently propagated to harvest $\mathrm{F}_{2}$ lines of PI $243518 \times$ 'Sloan'.

A total of $400 \mathrm{~F}_{2}$ lines were used in the stem cutting assay to phenotype their susceptibility to foliar chlorosis, foliar necrosis, and SDS foliar symptoms using an ordinal scale ranging from 0 to 10 (Fig. 1). The first trifoliate of each $F_{2}$ line was rated at five timepoints $(0,2,4,6$, and 8 days postinoculation) and the AUDPC was calculated. Because each $\mathrm{F}_{2}$ line produced only one first trifoliate for the stem cutting assay, there was no biological replicate to represent the same genotype. On the other hand, multiple biological replicates were included for the two parents throughout four independent phenotyping experiments. For the resistant parent PI $243518(n=31)$, the average \pm standard deviation AUDPC of foliar chlorosis, foliar necrosis, and SDS foliar symptoms was $19.32 \pm 5.77,9.61 \pm 7.15$, and $17.19 \pm 6.35$, respectively. For the susceptible parent 'Sloan' $(n=24)$, the average \pm standard deviation AUDPC of foliar chlorosis, foliar necrosis, and SDS foliar symptoms was $41.42 \pm 5.74,46.29 \pm 9.79$, and $49.71 \pm 6.74$, respectively. Based on these results, PI 243518 exhibited stronger resistance to foliar necrosis than foliar chlorosis.

Among the $400 \mathrm{~F}_{2}$ lines, significant pairwise correlations were found between foliar chlorosis, foliar necrosis, and SDS foliar symptoms. The distribution of the foliar chlorosis AUDPC was approximately normal, which indicates the potential of numerous QTLs with small effects contribute to the phenotypic difference in foliar chlorosis. On the other hand, the distributions of the foliar necrosis AUDPC and SDS foliar symptoms AUDPC were both deviated from normality, indicating the potential of a few QTLs with a large effect contribute to the phenotypic difference in foliar necrosis and SDS foliar symptoms. The correlation between the foliar necrosis AUDPC and the SDS foliar symptoms AUDPC was significant $(P<0.001)$ and the correlation coefficient was the highest among pairwise comparisons at 0.97, whereas the correlation coefficients between the foliar chlorosis AUDPC and either the foliar necrosis AUDPC or SDS foliar symptoms AUDPC were lower at around 0.5 (Fig. 2A). In summary, the $F_{2}$ population exhibited substantial phenotypic variations and symptomatic segregation for linkage mapping.

GBS to acquire SNPs in the $\mathrm{F}_{2}$ population of resistant $\mathrm{PI}$ 243518 and susceptible 'Sloan'. GBS and SNP calling using the TASSEL version 5 GBS pipeline version 2 identified 68,946 raw SNPs based on 'Williams82' genome assembly version 4; 7,372 of these 68,946 raw SNPs appeared to be polymorphic between the parents (PI 243518 and 'Sloan'), whereas 61,574 SNPs appeared to be monomorphic. Among the 7,372 polymorphic SNPs, 7,293 SNPs were mapped to one of the 20 chromosomes of soybean, whereas 79 SNPs were assigned to different scaffolds. Therefore, the 7,293 polymorphic SNPs were subsequently examined for segregation distortion and SNPs having significant segregation distortion were excluded, resulting in 1,454 SNPs remaining for downstream quality controls. A large proportion of missing genotypes and a clear batch effect were observed among the 1,454 SNPs (Fig. 3A), probably owing to technical variations generated in four independent experiments for DNA extractions and/or GBS library constructions. $\mathrm{F}_{2}$ lines processed in the first and second experiments showed lower missing genotypes compared with the third and fourth experiments. To exclude low-quality $\mathrm{F}_{2}$ lines and SNPs, additional quality controls were performed, including a serial removal of $\mathrm{F}_{2}$ progeny lines containing $>20 \%$ missing SNPs, which resulted in $138 \mathrm{~F}_{2}$ lines containing 1,454 SNPs (Fig. 3B). The removal of SNPs absent from $>20 \%$ of $\mathrm{F}_{2}$ progeny lines resulted in $138 \mathrm{~F}_{2}$ lines containing 1,166 SNPs (Fig. 3C). Manual removal of three $\mathrm{F}_{2}$ lines with relatively lower SNP density resulted in $135 \mathrm{~F}_{2}$ lines containing 1,166 SNPs (Fig. 3D). The final removal of duplicated SNPs resulted in $135 \mathrm{~F}_{2}$ lines containing 990
A

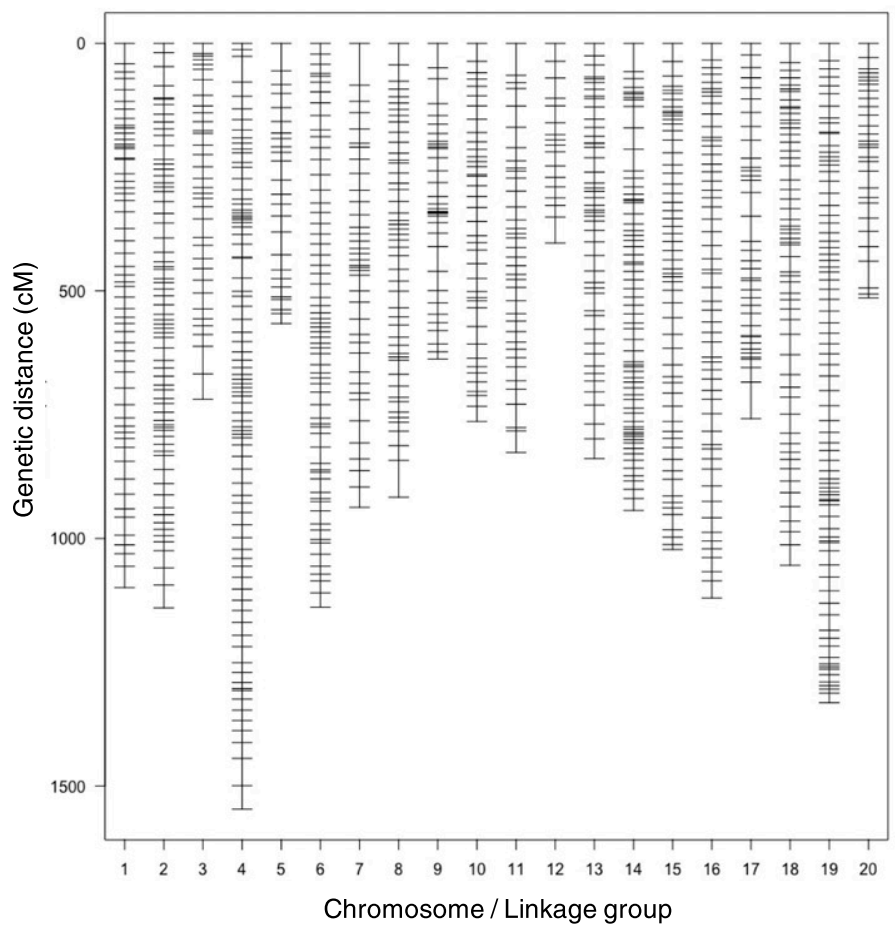

B

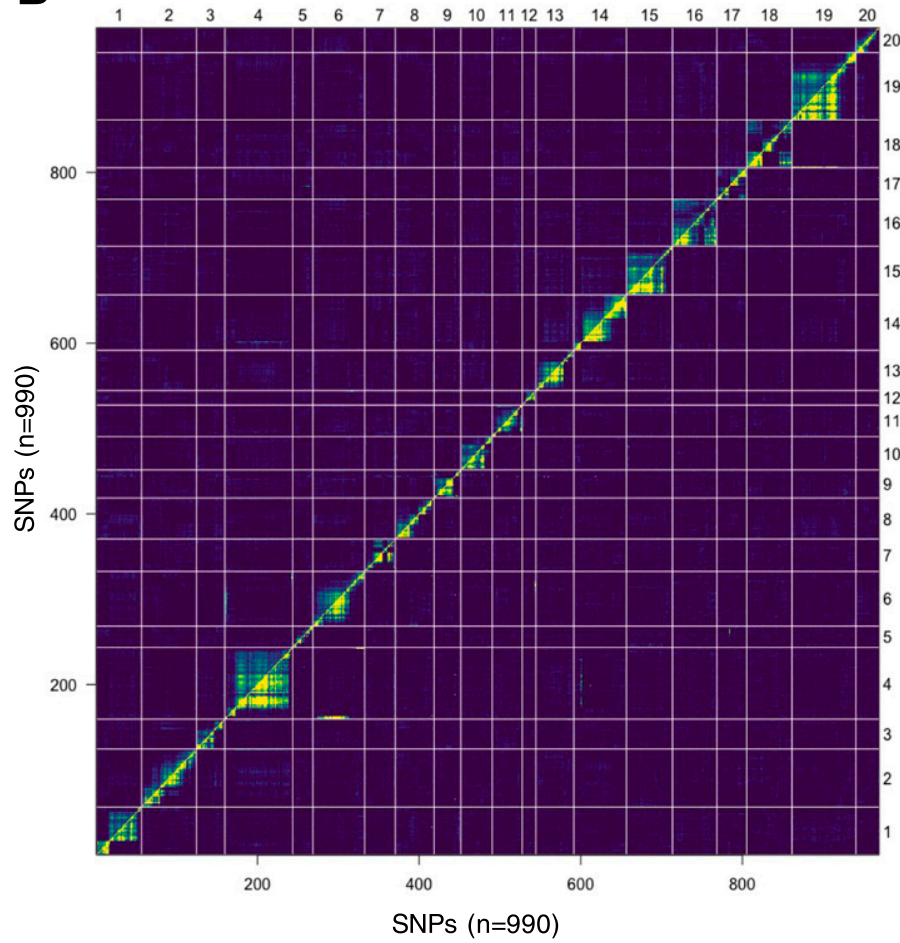

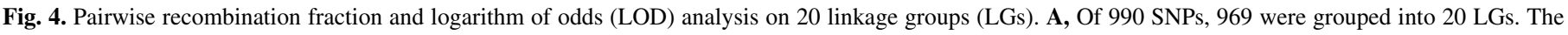

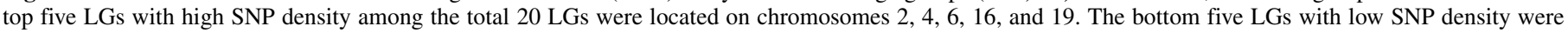

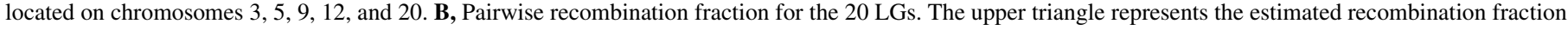

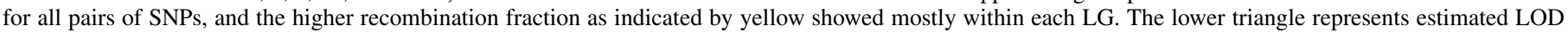

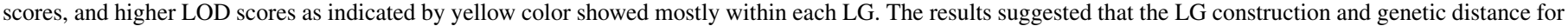
the 969 SNPs were suitable for linkage mapping. 
high-quality SNPs (Fig. 3E). The quality controls altogether downsized the mapping population from 400 to 135 lines and from 1,454 to 990 SNPs (Fig. 3F).

The phenotypic distributions of the foliar chlorosis AUDPC, foliar necrosis AUDPC, and SDS foliar symptoms AUDPC in these quality-controlled $135 \mathrm{~F}_{2}$ lines were compared with the original 400 $\mathrm{F}_{2}$ lines. The downsizing of the mapping population from 400 to 135 $\mathrm{F}_{2}$ lines did not evidently alter the phenotypic distributions, but it caused higher correlation between the foliar chlorosis AUDPC and the foliar necrosis AUDPC and between the foliar chlorosis AUDPC and the SDS foliar symptoms AUDPC (Fig. 2B). In addition, 969 of the 990 SNPs were grouped into LGs by MSTmap software (Fig.
4A), and the 20 LGs were estimated for their pairwise recombination fraction to verify the absence of abnormal crossing events among LGs (Fig. 4B). The results identified a total of 969 high-quality and high-confidence SNPs forming 20 LGs from the GBS approach and TASSEL GBS pipeline version 2, and this dataset was used in linkage mapping.

Linkage mapping for the foliar chlorosis AUDPC, foliar necrosis AUDPC, and SDS foliar symptoms AUDPC in the $F_{2}$ population of resistant $P I 243518$ and susceptible 'Sloan'. Linkage mapping for the foliar chlorosis AUDPC did not identify any significant peak. The strongest signal was detected on chromosome 3 (Fig. 5A) but the logarithm of odds (LOD) score

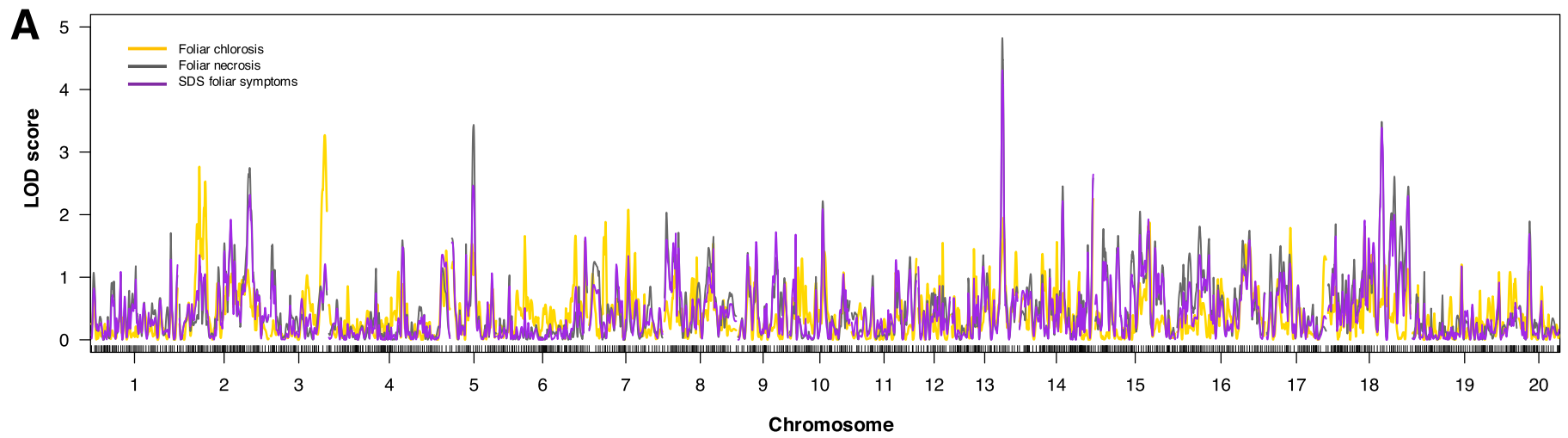

B

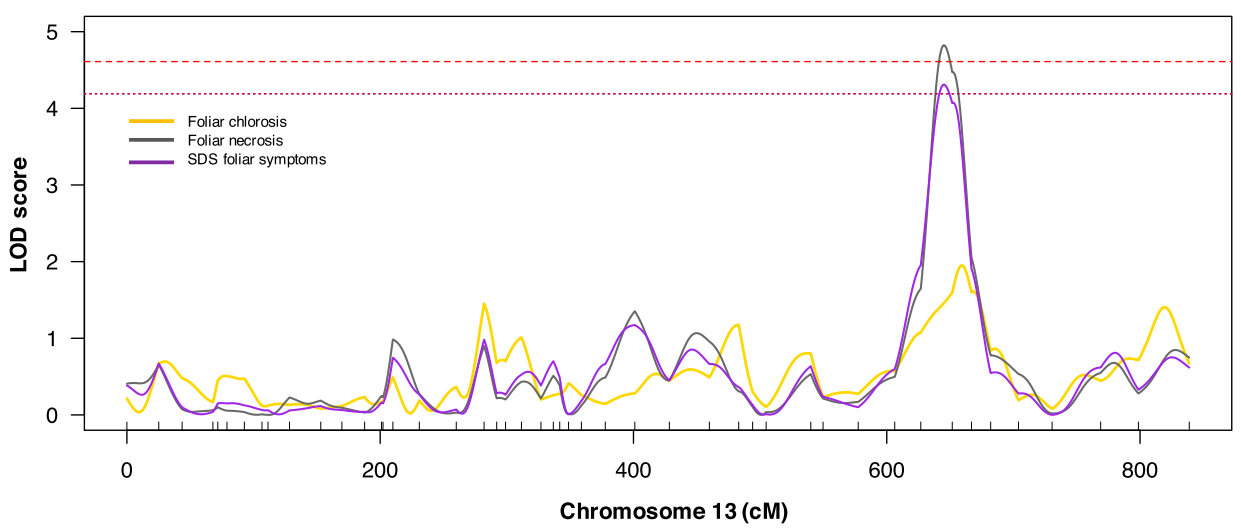

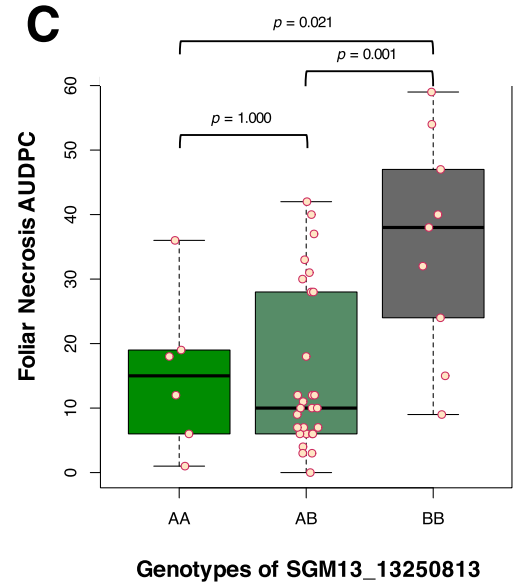
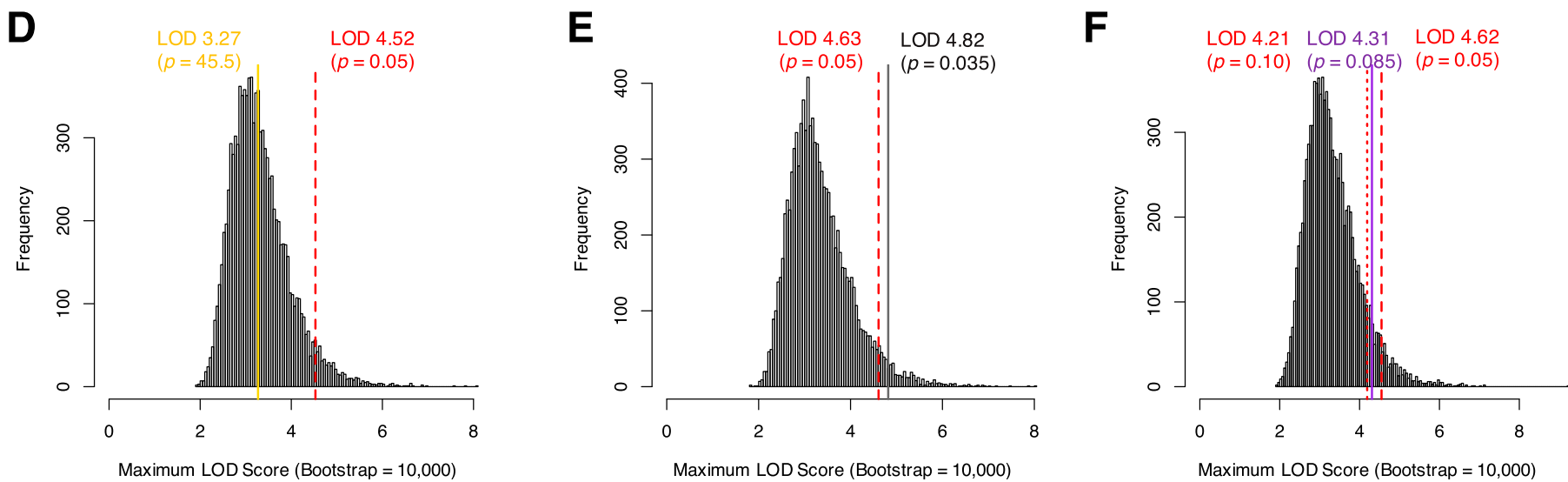

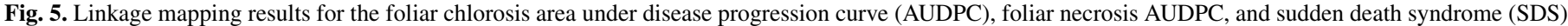

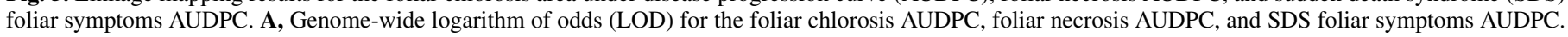

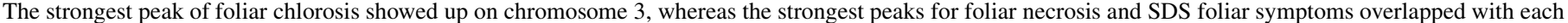

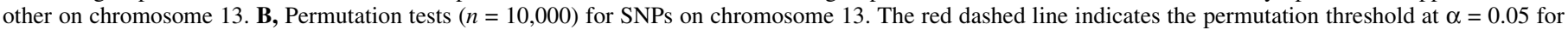

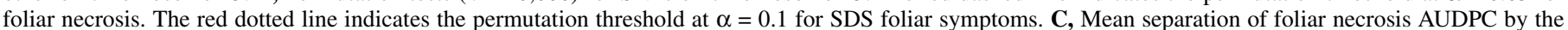

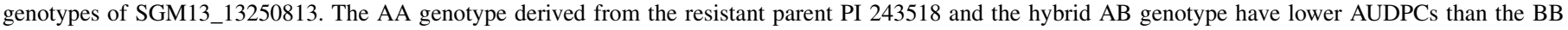

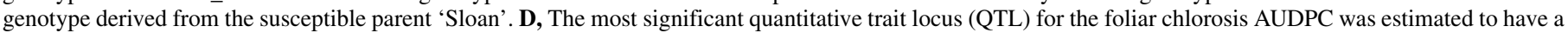

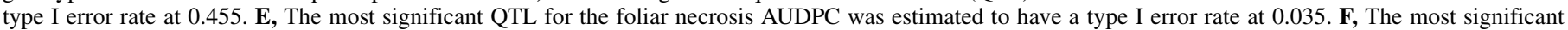
QTL for the SDS foliar symptoms AUDPC was the same peak to foliar necrosis AUDPC, which was estimated to have a type I error rate at 0.085. 
was estimated to have $45.5 \%$ probability of making a type I error after the permutation test with 10,000 iterations (Fig. 5D). On the other hand, a peak on chromosome 13 was detected for both the foliar necrosis AUDPC and the SDS foliar symptoms AUDPC (Fig. $5 \mathrm{~A}$ and $\mathrm{B}$ ), and the LOD scores were estimated to have 3.5 and $8.5 \%$ probability of making a type I error, respectively (Fig. 5E and F). The $95 \%$ confidence interval of this peak was estimated to locate between 638 to $658 \mathrm{cM}$, which is close to the physical position between 7.84 to $20.12 \mathrm{Mbp}$ on chromosome 13 of soybean Williams82 genome assembly version 4 (Table 1). The peak was centered at $645 \mathrm{cM}$, and the closest associated SNP was SGM13_ 13250813. Using this SNP for mean separation analysis, the AA genotype (homozygous for the resistant parent PI 243518) and the hybrid $\mathrm{AB}$ genotype were not significantly different from each other $(P=1.000)$, but both genotypes were significantly different from the BB genotype (homozygous for the susceptible parent 'Sloan'; $P=$ 0.021 and $P=0.001$, respectively) (Fig. 5C). The additive effect of the $A$ allele was estimated to be less significant compared with the dominant effect of the $A$ allele, which was significant for both the foliar necrosis AUDPC and the SDS foliar symptoms AUDPC (Table 2). Therefore, the linkage mapping results identified a dominant peak on chromosome 13 for the foliar necrosis AUDPC, and this peak might also contribute to the overall resistance to SDS foliar symptoms.

\section{DISCUSSION}

Identifying SDS resistance and incorporating it into elite lines has been the goal of soybean pathologists and breeders for many years. Although strides have been made in the commercial varieties for SDS resistance, advanced exploring and understanding SDS resistance is desired. Given the low frequency of SDS resistance observed in the current USDA soybean germplasm collection (Mueller et al. 2002), the gathering and screening of additional germplasm lines may likely discover limited resistance sources. An alternative approach to identifying SDS resistance is to re-evaluate current germplasm lines using more precise phenotyping methods to resolve the underlying genetics. Most SDS resistance screening and mapping studies evaluate foliar symptoms in their entirety in the field, which should be considered as the combined outcome of root resistance and foliar resistance. Studies have discovered more than 100 QTLs and numerous SNPs; however, there is the potential concern of interference by the root responses to the soybean cyst nematode (SCN) (Chang et al. 2018). To date, few studies have phenotyped germplasm lines using $F$. virguliforme phytotoxic culture filtrates to exclude the interference of SCN resistance and to specifically screen for foliar resistance. One study screened germplasm lines using a Soybean mosaic virus (SMV)-mediated transit overexpression of a phytotoxic effector FvNIS1, which reported SNPs on chromosomes 6 and 20 (Chang et al. 2016a). Other studies phenotyped two biparental populations initially using the phytotoxic $F$. virguliforme culture filtrates and later reexamined the same population using a greenhouse screen (Swaminathan et al. 2016, 2018). By comparing results from the two screening methods on the same population, these two studies provide higher confidence for overlapping SDS resistance regions including chromosomes 9 and 20. In another study, resistance screening was not only performed using $F$. virguliforme phytotoxic culture filtrates, but it was also evaluated for foliar chlorosis specifically. The integration of linkage mapping, GWASs, and genomic synteny enabled the discovery of a causal mutated genotype (dldld2d2), the STAY-GREEN gene with the loss-ofsusceptibility mechanism, as one of the resistance mechanisms for foliar chlorosis (Chang et al. 2019). Similarly, a symptom-specific phenotyping strategy was applied in this study to phenotype an $\mathrm{F}_{2}$ population from a cross of the susceptible variety Sloan and the germplasm line PI 243518, which exhibits resistance to foliar chlorosis, foliar necrosis, and overall SDS foliar symptoms. PI 243518 is also the only germplasm line that has the genotype (D1D1D2D2) of STAY-GREEN genes but is capable of exhibiting

TABLE 1. Statistics of the significant quantitative trait locus identified from $135 \mathrm{~F}_{2}$ population of PI $243518 \times$ 'Sloan'

\begin{tabular}{lcll}
\hline Phenotype & Peak (cM/LOD score) $)^{\mathrm{a}}$ & $\begin{array}{c}\text { Bayes credible 95\% interval } \\
(\mathrm{cM} / \text { LOD score })\end{array}$ & Nearest marker (cM) \\
\hline \multirow{2}{*}{ SDS foliar necrosis } & \multirow{2}{*}{ 646/4.824 } & Left border: 638/4.101 & $\begin{array}{l}\text { SGM13_20122322: 626.856 } \\
\text { SGM13_13250813: 651.712 } \\
\text { SGM13_7836748: 666.906 }\end{array}$ \\
\hline
\end{tabular}

a $\mathrm{LOD}=$ logarithm of the odds, $R^{2}=$ coefficient of determination (the proportion of variance in SDS foliar necrosis explained by this locus), and SDS = sudden death syndrome.

TABLE 2. Additive and dominant effect of the single nucleotide polymorphism SGM13_13250813 in the locus identified from $135 \mathrm{~F}_{2}$ population of PI $243518 \times$ 'Sloan'

\begin{tabular}{|c|c|c|c|c|}
\hline Phenotype $^{a}$ & Additive effect & $P$ value & Dominant effect & $P$ value \\
\hline SDS foliar chlorosis AUDPC & -0.1072 & 0.2954 & -0.2923 & 0.1115 \\
\hline SDS foliar necrosis AUDPC & -0.4592 & 0.0473 & -1.2562 & 0.0013 \\
\hline SDS foliar symptoms AUDPC & -0.2712 & 0.0747 & -0.7807 & 0.0030 \\
\hline
\end{tabular}

a SDS = sudden death syndrome and AUDPC = area under disease progression curve.

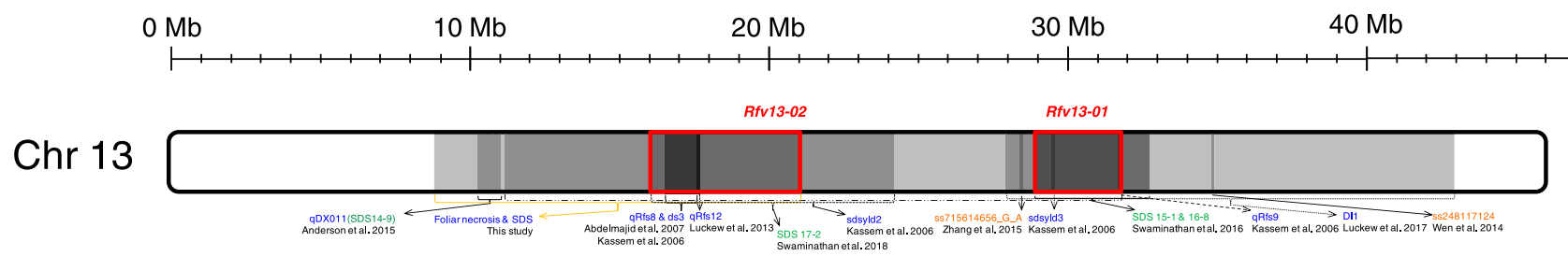

Fig. 6. A schematic illustration for the Rfv13-02 (resistance to Fusarium virguliforme) locus. The yellow line indicates the quantitative trait loci (QTL) identified in this study, and the red blocks highlight the reproducible regions on chromosome 13 (Chr 13) by at least three different studies. 
resistance to foliar chlorosis. Unfortunately, there was no significant QTL for foliar chlorosis identified in this study. This result could be attributable to the quantitative nature of foliar chlorosis resistance being governed by many loci with minor effects. An RIL population with lower heterozygosity and larger population size may increase the mapping power to resolve the genetics of PI 243518 resistance to foliar chlorosis.

This study identified a novel region (about 8.76 to $21.00 \mathrm{Mbp}$ based on Williams82 genome version a2.v1) on chromosome 13 associated with resistance to foliar necrosis and SDS foliar symptoms, which harbors several previously reported QTLs, including SDS 14-9 (a.k.a. qDX011) (Anderson et al. 2015), qRfs 8 (Abdelmajid et al. 2007), $d s 3$ (Kassem et al. 2006), $q R f s 12$ (Luckew et al. 2013), SDS 17-2 (Swaminathan et al. 2018), and sdsyld2 (Kassem et al. 2006). In addition, two SSR markers, Satt160 and Satt252, were also reported to associate with this locus (Abdelmajid et al. 2007; Luckew et al. 2013). Because the intersection, from the left end of SDS 17-2 to the right end of the locus identified in this study (about 15.98 to $21.00 \mathrm{Mbp}$ ), has been reported independently by three mapping studies (this study; Kassem et al. 2006; Swaminathan et al. 2018), a new nomenclature Rfv13-02 is recommended for this reproducible locus (Fig. 6). Nonetheless, the range of $R f v 13-02$ is still too wide to target a particular candidate resistance gene. Fine mapping is required to map the interval, identify the resistance genes, and understand the molecular mechanisms of Rfv13-02.

Worth noting is that unlike many SDS resistance mapping studies that ended up with a signal on chromosome 18 near the SCN resistance locus Rhgl, this study as well as most studies using F. virguliforme phytotoxic culture filtrate did not identify QTLs close to the $R h g l$ region. It is possible that $R f v 18-01$ exists only in the genetic background of some partially resistant varieties such as 'Forrest'; alternatively, the results of this study might be another case to support the confounding effect between $R f v 18-01$ and $R h g 1$ owing to the co-occurrence of SDS and SCN in fields (Chang et al. 2018). Based on the USDA GRIN database, PI 243518 exhibits moderate susceptibility to SCN race 3 and race 4; therefore, PI 243518 may be a pure genetic source for studying SDS foliar resistance without the interference of Rhgl. Accordingly, phenotyping PI 243518 and its RILs in SCN-infested fields would show whether these lines still exhibit SDS foliar resistance when roots are infected by SCN, and such an observation would be evidence to support that $\mathrm{SCN}$ infection in roots does not synergistically promote the severity of SDS foliar symptoms.

Although there was only one QTL found for foliar necrosis and SDS foliar symptoms in this study, it is possible that there are more resistance sources in PI 243518 as this accession exhibits clear phenotypic resistance to foliar chlorosis, foliar necrosis, and overall SDS foliar symptoms. Further development of the $\mathrm{F}_{2}$ lines into an RIL population with lower heterozygosity and higher recombination events may not only allow the detection of more SNPs but also break down the linkage interval to identify resistance candidate genes. In addition, better GBS practices using a high-quality DNA extraction, a double restriction enzyme digestion protocol, and/or other practices in the GBS library construction may reduce batch effects and acquire more SNPs in the RIL population.

\section{ACKNOWLEDGMENTS}

We thank Yuya Liang (Department of Soil and Crop Sciences, Texas A\&M University) for her suggestions on linkage construction.

\section{LITERATURE CITED}

Abdelmajid, K. M., Meksem, K., Wood, A. J., and Lightfoot, D. A. 2007. Loci underlying SDS and SCN resistance mapped in the 'Essex' by 'Forrest' soybean recombinant inbred lines. Rev. Biol. Biotechnol. 6:2-10.

Abdelmajid, K. M., Ramos, L., Leandro, L. F., Mbofung, G., Hyten, D. L., Kantartzi, S. K., et al. 2012. The 'PI 438489B' by 'Hamilton' SNP-based genetic linkage map of soybean (Glycine $\max$ (L.) Merr.) identified quantitative trait loci that underlie seedling SDS resistance. J. Plant Genome Sci. $1: 18-30$.

Allen, T. W., Bradley, C. A., Sisson, A. J., Byamukama, E., Chilvers, M. I., Coker, C. M., et al. 2017. Soybean yield loss estimates due to diseases in the United States and Ontario, Canada, from 2010 to 2014. Plant Health Prog. 18:19-27.

Anderson, J., Akond, M., Kassem, M. A., Meksem, K., and Kantartzi, S. K. 2015. Quantitative trait loci underlying resistance to sudden death syndrome (SDS) in MD96-5722 by 'Spencer' recombinant inbred line population of soybean. 3 Biotech. 5:203-210.

Bradbury, P. J., Zhang, Z., Kroon, D. E., Casstevens, T. M., Ramdoss, Y., and Buckler, E. S. 2007. TASSEL: Software for association mapping of complex traits in diverse samples. Bioinformatics 23:2633-2635.

Broman, K. W., Gatti, D. M., Simecek, P., Furlotte, N. A., Prins, P., Sen, Ś., et al. 2019. R/qt12: Software for mapping quantitative trait loci with highdimensional data and multiparent populations. Genetics 211:495-502.

Broman, K. W., Wu, H., Sen, Ś., and Churchill, G. A. 2003. R/qtl: QTL mapping in experimental crosses. Bioinformatics 19:889-890.

Brzostowski, L. F., Pruski, T. I., Hartman, G. L., Bond, J. P., Wang, D., Cianzio, S. R., et al. 2018. Field evaluation of three sources of genetic resistance to sudden death syndrome of soybean. Theor. Appl. Genet. 131: 1541-1552.

Chang, H.-X., Domier, L. L., Radwan, O., Yendrek, C. R., Hudson, M. E., and Hartman, G. L. 2016a. Identification of multiple phytotoxins produced by Fusarium virguliforme including a phytotoxic effector (FvNIS1) associated with sudden death syndrome foliar symptoms. Mol. Plant-Microbe Interact. 29:96-108.

Chang, H.-X., Lipka, A. E., Domier, L. L., and Hartman, G. L. 2016b. Characterization of disease resistance loci in the USDA soybean germplasm collection using genome-wide association studies. Phytopathology 106: 1139-1151.

Chang, H.-X., Roth, M. G., Wang, D., Cianzio, S. R., Lightfoot, D. A., Hartman, G. L., et al. 2018. Integration of sudden death syndrome resistance loci in the soybean genome. Theor. Appl. Genet. 131: 757-773.

Chang, H.-X., Tan, R., Hartman, G. L., Wen, Z., Sang, H., Domier, L. L., et al. 2019. Characterization of soybean STAY-GREEN genes in susceptibility to foliar chlorosis of sudden death syndrome. Plant Physiol. 180:711-717.

Chang, S. J. C., Doubler, T. W., Kilo, V., Suttner, R., Klein, J., Schmidt, M. E., et al. 1996. Two additional loci underlying durable field resistance to soybean sudden death syndrome (SDS). Crop Sci. 36:1684-1688.

Elshire, R. J., Glaubitz, J. C., Sun, Q., Poland, J. A., Kawamoto, K., et al. 2011. A robust, simple genotyping-by-sequencing (GBS) approach for high diversity species. PLoS One 6:e19379.

Feenstra, B., Skovgaard, I. M., and Broman, K. W. 2006. Mapping quantitative trait loci by an extension of the Haley-Knott regression method using estimating equations. Genetics 173:2269-2282.

Hartman, G. L., Chang, H.-X., and Leandro, L. F. 2015. Research advances and management of soybean sudden death syndrome. Crop Prot. 73:60-66.

Herrmann, I., Vosberg, S. K., Ravindran, P., Singh, A., Chang, H.-X., Chilvers, M. I., et al. 2018. Leaf and canopy level detection of Fusarium virguliforme (sudden death syndrome) in soybean. Remote Sens. 10:426.

Hnetkovsky, N., Chang, S. J. C., Doubler, T. W., Gibson, P. T., and Lightfoot, D. A. 1996. Genetic mapping of loci underlying field resistance to soybean sudden death syndrome (SDS). Crop Sci. 36:393-400.

Iqbal, M. J., Meksem, K., Njiti, V. N., Kassem, M. A., and Lightfoot, D. A. 2001. Microsatellite markers identify three additional quantitative trait loci for resistance to soybean sudden-death syndrome (SDS) in Essex $\times$ Forrest RILs. Theor. Appl. Genet. 102:187-192.

Kandel, Y. R., Bradley, C. A., Chilvers, M. I., Mathew, F. M., Tenuta, A. U., Smith, D. L., et al. 2019a. Effect of seed treatment and foliar crop protection products on sudden death syndrome and yield of soybean. Plant Dis. 103:1712-1720.

Kandel, Y. R., Leandro, L. F. S., and Mueller, D. S. 2019b. Effect of tillage and cultivar on plant population, sudden death syndrome, and yield of soybean in Iowa. Plant Health Prog. 20:29-34.

Kandel, Y. R., Wise, K. A., Bradley, C. A., Chilvers, M. I., Tenuta, A. U., and Mueller, D. S. 2016a. Fungicide and cultivar effects on sudden death syndrome and yield of soybean. Plant Dis. 100:1339-1350.

Kandel, Y. R., Wise, K. A., Bradley, C. A., Tenuta, A. U., and Mueller, D. S. 2016b. Effect of planting date, seed treatment, and cultivar on plant population, sudden death syndrome, and yield of soybean. Plant Dis. 100:1735-1743.

Kassem, M. A., Shultz, J., Meksem, K., Cho, Y., Wood, A. J., Iqbal, M. J., et al. 2006. An updated 'Essex' by 'Forrest' linkage map and first composite interval map of QTL underlying six soybean traits. Theor. Appl. Genet. 113:1015-1026.

Kazi, S., Shultz, J., Afzal, J., Johnson, J., Njiti, V. N., and Lightfoot, D. A. 2008. Separate loci underlie resistance to root infection and leaf scorch 
during soybean sudden death syndrome. Theor. Appl. Genet. 116: 967-977.

Koenning, S. R., and Wrather, J. A. 2010. Suppression of soybean yield potential in the continental United States by plant diseases from 2006 to 2009. Online publication. Plant Health Prog. 11. doi:10.1094/PHP2010-1122-01-RS.

Luckew, A. S., Leandro, L. F., Bhattacharyya, M. K., Nordman, D. J., Lightfoot, D. A., and Cianzio, S. R. 2013. Usefulness of 10 genomic regions in soybean associated with sudden death syndrome resistance. Theor. Appl. Genet. 126:2391-2403.

Luckew, A. S., Swaminathan, S., Leandro, L. F., Orf, J. H., and Cianzio, S. R. 2017. 'MN1606SP' by 'Spencer' filial soybean population reveals novel quantitative trait loci and interactions among loci conditioning SDS resistance. Theor. Appl. Genet. 130:2139-2149.

Madden, L. V., Hughes, G., and van den Bosch, F. 2007. The Study of Plant Disease Epidemics. American Phytopathological Society, St. Paul, MN.

Martin, M. 2011. Cutadapt removes adapter sequences from high-throughput sequencing reads. EMBnet J. 17:10-12.

Meksem, K., Doubler, T. W., Chancharoenchai, K., Njiti, V. N., Chang, S. J. C., Arelli, A. P. R., et al. 1999. Clustering among loci underlying soybean resistance to Fusarium solani, SDS and SCN in near-isogenic lines. Theor. Appl. Genet. 99:1131-1142.

Mueller, D. S., Hartman, G. L., Nelson, R. L., and Pedersen, W. L. 2002. Evaluation of Glycine max germ plasm for resistance to Fusarium solani $\mathrm{f}$. sp. glycines. Plant Dis. 86:741-746.

Navi, S. S., and Yang, X. B. 2016. Sudden death syndrome-a growing threat of losses in soybeans. CAB Rev. 11:1-13.

Njiti, V. N., Meksem, K., Iqbal, M. J., Johnson, J. E., and Kassem, M. A., Zobrist, K. F., et al. 2002. Common loci underlie field resistance to soybean sudden death syndrome in 'Forrest', 'Pyramid', 'Essex', and 'Douglas'. Theor. Appl. Genet. 104:294-300.

Prabhu, R. R., Njiti, V. N., Bell-Johnson, B., Johnson, J. E., Schmidt, M. E., Klein, J. H., et al. 1999. Selecting soybean cultivars for dual resistance to soybean cyst nematode and sudden death syndrome using two DNA markers. Crop Sci. 39:982-987.

Roth, M. G., Noel, Z. A., Wang, J., Warner, F., Byrne, A. M., and Chilvers, M. I. 2019. Predicting soybean yield and sudden death syndrome development using at-planting risk factors. Phytopathology 109:17101719 .
Swaminathan, S., Abeysekara, N. S., Knight, J. M., Liu, M., Dong, J., Hudson, M. E., et al. 2018. Mapping of new quantitative trait loci for sudden death syndrome and soybean cyst nematode resistance in two soybean populations. Theor. Appl. Genet. 131:1047-1062.

Swaminathan, S., Abeysekara, N. S., Liu, M., Cianzio, S. R., and Bhattacharyya, M. K. 2016. Quantitative trait loci underlying host responses of soybean to Fusarium virguliforme toxins that cause foliar sudden death syndrome. Theor. Appl. Genet. 129:495-506.

Tan, R., Collins, P. J., Wang, J., Wen, Z., Boyse, J. F., Laurenz, R. G., et al. 2019. Different loci associated with root and foliar resistance to sudden death syndrome (Fusarium virguliforme) in soybean. Theor. Appl. Genet. 132:501-513.

Tan, R., Serven, B., Collins, P. J., Zhang, Z., Wen, Z., Boyse, J. F., et al. 2018. QTL mapping and epistatic interaction analysis of field resistance to sudden death syndrome (Fusarium virguliforme) in soybean. Theor. Appl. Genet. 131:1729-1740.

Wen, Z., Tan, R., Yuan, J., Bales, C., Du, W., Zhang, S., et al. 2014. Genomewide association mapping of quantitative resistance to sudden death syndrome in soybean. BMC Genomics 15:809.

Wickland, D. P., Battu, G., Hudson, K. A., Diers, B. W., and Hudson, M. E. 2017. A comparison of genotyping-by-sequencing analysis methods on low-coverage crop datasets shows advantages of a new workflow, GB-eaSy. BMC Bioinformatics 18:586.

Wrather, J. A., and Koenning, S. R. 2009. Effects of diseases on soybean yields in the United States 1996 to 2007. Plant Health Prog. 10. doi: 10.1094/PHP-2009-0401-01-RS.

Wu, Y., Bhat, P. R., Close, T. J., and Lonardi, S. 2008. Efficient and accurate construction of genetic linkage maps from the minimum spanning tree of a graph. PLoS Genet. 4:e1000212.

Xiang, Y., Scandiani, M. M., Herman, T. K., and Hartman, G. L. 2015. Optimizing conditions of a cell-free toxic filtrate stem cutting assay to evaluate soybean genotype responses to Fusarium species that cause sudden death syndrome. Plant Dis. 99:502-507.

Yuan, J., Bashir, R., Salas, G., Sharma, H., Srour, A., and Lightfoot, D. A. 2012. New approaches to selecting resistance or tolerance to SDS and Fusarium root rot. J. Plant Genome Sci. 1:10-17.

Zhang, J., Singh, A., Mueller, D. S., and Singh, A. K. 2015. Genome-wide association and epistasis studies unravel the genetic architecture of sudden death syndrome resistance in soybean. Plant J. 84:1124-1136. 